\title{
A Prospective Longitudinal Study to Estimate the "Adjusted Cortisol Percentile" in Preterm Infants
}

\author{
PAK C. NG, SAM P. S. WONG, IRIS H. S. CHAN, HUGH S. LAM, CHEUK H. LEE, AND CHRISTOPER W. K. LAM
}

Departments of Pediatrics [P.C.N., H.S.L., C.H.L.], Statistics [S.P.S.W.], Chemical Pathology [I.H.S.C., C.W.K.L.], Prince of Wales

Hospital, The Chinese University of Hong Kong, New Territories, Hong Kong

\begin{abstract}
The normal range of serum cortisol concentrations and the appropriate levels of circulating cortisol in different clinical situations in preterm infants are not well defined. This study aimed to evaluate the impact of perinatal factors on circulating cortisol levels in preterm infants and to create a quantitative model that could estimate the "adjusted cortisol percentile." Serial serum cortisol concentrations were measured in 209 infants $\leq 32$ wk gestation on d $1,4,7,14$, and 21 of life. Seven perinatal factors or conditions that could affect circulating cortisol level were identified. Serum cortisol levels were higher on $\mathrm{d} 4(p=0.007)$ and $\mathrm{d} 7(p=0.007)$ but lower on d $21(p=0.001)$ compared with d 1. Serum cortisol was also higher in infants on nasal continuous positive airway pressure $(p=0.003)$; requiring a second vasopressor $(p<0.001)$; with intraventricular hemorrhage ( $\geq$ grade $3 ; p<0.001$ ); with histologic chorioamnionitis $(p=0.007)$; with severe lung disease ( $p=$ $0.046)$; and with decreasing GA $(p<0.001)$. A mathematical equation was proposed based on factors derived in this preliminary study for estimating the adjusted cortisol percentile. Frontline neonatologists could now access the equation on our Web site (http://www.sta.cuhk.edu.hk/pswong/ACortP.html) to calculate the adjusted cortisol percentile, which could potentially improve the interpretation of circulating cortisol in different clinical situations. (Pediatr Res 69: 511-516, 2011)
\end{abstract}

$\mathrm{N}^{\circ}$ ormal development of the hypothalamic-pituitary-adrenal (HPA) axis is essential for regulation of intrauterine homeostasis, timely maturation and differentiation of vital organ systems, and postnatal survival of newborns under stressful conditions $(1,2)$. Over the past two decades, many reports have emphasized the importance of associations between circulating cortisol and systemic hypotension (3-5), bronchopulmonary dysplasia (BPD) (6-8), and other stressful complications (9) in preterm and very LBW (VLBW) infants. It has been postulated that inadequate and low serum cortisol in these infants may be due to transient adrenal insufficiency of prematurity (i.e. "immature" intermediate enzyme systems in the steroidogenesis pathway resulting in insufficient cortisol production and with rapid recovery of the hormonal axis in early postnatal life) $(5,10-13)$ or relative adrenal insufficiency

Received June 22, 2010; accepted December 11, 2010.

Correspondence: Professor Pak C. Ng, MD, Department of Pediatrics, Prince of Wales Hospital, 6th Floor, Clinical Sciences Building, Shatin, New Territories, Hong Kong; e-mail: pakcheungng@cuhk.edu.hk

Supported by the Research Fund of the Department of Pediatrics, The Chinese University of Hong Kong.

Supplemental digital content is available for this article. Direct URL citations appear in the printed text and are provided in the HTML and PDF versions of this article on the journal's Web site (www.pedresearch.org). (i.e. failure to produce "appropriate quantity" of cortisol in response to stressful situations compared with the normal healthy subject of the same gestational and postnatal age) $(6,8,11)$. The use of physiologic or therapeutic doses of systemic corticosteroids may be beneficial in these situations $(3,4)$. However, the normal range of serum cortisol or what is considered to be the appropriate level of circulating cortisol in preterm infants is not known (14). More importantly, the effects of specific intrinsic (e.g. GA or postnatal age) and extrinsic factors (e.g. disease processes or treatment modalities) on the HPA axis have not been systematically evaluated (14). In the absence of such knowledge, meaningful interpretation of cortisol data is not possible. Furthermore, the use of systemic corticosteroids in infants with relatively normal or high levels of cortisol may expose the patients to unnecessary risks such as spontaneous intestinal perforation and should therefore be avoided. Previous studies investigating the response of the HPA axis to stress and serum cortisol in the postnatal period have many limitations. Some studies concentrated mainly on ventilated preterm infants $(9,10)$, whereas the others monitored serum cortisol at only one or two timepoints $(4,5,9,10)$ or performed the analysis of results as a secondary outcome of the original objective (9). Recently, we have also observed that a proportion of asymptomatic and unstressed VLBW infants who did not require mechanical ventilation and vasopressor support had very low circulating levels of cortisol, often $<100 \mathrm{nmol} / \mathrm{L}(<3.62 \mu \mathrm{g} / \mathrm{dL}$; our own neonatal unit audit). This suggests that even very low serum cortisol levels may be adequate for maintaining circulatory and metabolic homeostasis in "healthy" and unstressed preterm neonates. In this study, we aimed to longitudinally monitor the serum cortisol concentrations at preselected timepoints in a cohort of preterm infants $\leq 32 \mathrm{wk}$ gestation born within a 50-mo period. The objectives were i) to identify and to assess the magnitude of influence of significant stressful events or conditions, including the effect of postnatal age, on the circulating cortisol level, and ii) to develop a quantitative mathematical model for working out the adjusted percentile of circulating cortisol, taking into account all identified significant stressful factors.

\footnotetext{
Abbreviations: AIC, Akaike information criterion; BIC, Bayesian information criterion; BPD, bronchopulmonary dysplasia; HFOV, high-frequency oscillatory ventilation; HPA, hypothalamic-pituitary-adrenal; IPPV, intermittent positive pressure ventilation; IVH, intraventricular hemorrhage; nCPAP, nasal continuous positive airway pressure; PE, prediction errors; VLBW, very LBW
} 
Together with the clinical presentation, such a model would enable frontline neonatologists to assess the adequacy of circulating cortisol and may assist in deciding whether to use physiologic or therapeutic doses of corticosteroids in different clinical situations, but even more importantly, when to avoid using such treatment.

\section{MATERIALS AND METHODS}

Study population. A total of 209 preterm infants were prospectively enrolled in the study within a 50-mo period between January 2003 and July 2007 (excluding 4 mo during the outbreak of Severe Acute Respiratory Syndrome in Hong Kong). All preterm infants $\leq 32 \mathrm{wk}$ gestation were eligible for inclusion. Infants were excluded if they had chromosomal abnormalities, midline facial defects, or lethal congenital disorders. Blood samplings were discontinued only when parental permission was withdrawn, infants were commenced on postnatal systemic dexamethasone, infants had left the neonatal unit, or infants died during the study period (for details of the clinical management, see Supplemental Digital Content available at http://links.lww.com/PDR/XXX) (15-17).

Blood sample collection and hormone assays. Serial blood samples were collected at five timepoints: within the first $24 \mathrm{~h}(\mathrm{~d} 1)$ and on d 4, 7, 14, and 21 of life. The reasons for choosing this time sequence were based on the following rationale: i) substantial remodeling of the adrenal cortex occurs soon after birth $(1,2)$, ii) previous observations suggested that adrenal insufficiency of prematurity with refractory hypotension occurred most frequently within the first $7 \mathrm{~d}$ of life $(3,4)$, iii) our previous studies using the human corticotropin stimulation test for assessing pituitary and adrenal reserves at $\mathrm{d}$ 7 and $\mathrm{d} 14(5,10,18)$ did not provide information of serum cortisol levels at the most vulnerable period within the first week of life, and iv) serial monitoring of serum cortisol, in particular, after the second week could provide valuable insights about the adaptation of the HPA axis to extrauterine life. Blood samples $(0.5 \mathrm{~mL})$ were collected from an indwelling arterial line or by venipuncture in the morning between 0800 and $1000 \mathrm{~h}$ and coincided exactly with the timing of routine blood sampling for blood counts, blood gases, electrolytes, or renal profile, so as to minimize the disturbance to these infants. All specimens were immediately immersed in ice and transported to the laboratory for processing. Serum cortisol concentrations were measured by electrochemiluminescence immunoassay (E170 Modular Analytics; Roche Diagnostics Corp, Indianapolis, IN). The sensitivity and interassay coefficients of variation were $<8.5 \mathrm{nmol} / \mathrm{L}(<0.31 \mu \mathrm{g} / \mathrm{dL})$, and $5.4 \%$ at $104 \mathrm{nmol} / \mathrm{L}$ $(3.77 \mu \mathrm{g} / \mathrm{dL}), 3.6 \%$ at $581 \mathrm{nmol} / \mathrm{L}(21.05 \mu \mathrm{g} / \mathrm{dL})$, and $3.1 \%$ at $953 \mathrm{nmol} / \mathrm{L}$ $(34.52 \mu \mathrm{g} / \mathrm{dL})$. Serum cortisol concentration in nanomoles per liter can be converted to micrograms per deciliter by dividing by a factor of 27.6.

Data collection. The important demographic characteristics of study infants are presented in Table 1. Clinical data collected within the first $21 \mathrm{~d}$ of life on the cardiovascular, respiratory, and other organ systems are summarized in Table 2 (details of clinical management are available at http://links.lww.com/PDR/XXX).

Statistical analyses. The descriptive statistics on demographic data, treatments, and outcomes are expressed as median (interquartile range) or number (percentage). As longitudinal measurements were obtained for each infant, the mixed-effects models (19) were used to assess the associations between serum cortisol and gestational/postnatal age, birth weight, important physiologic or environmental factors, treatments, and outcomes (i.e. all parameters in Tables 1 and 2, list of parameters, available at http://links.lww.com/PDR/XXX). The mixed-effects models were used to adjust for the individual longitudinal random factor and the covariates were chosen by a stepwise selection procedure that consisted of a "forward addition" step and a "backward elimination" step. Both steps were guided by the goodness-of-fit criterionAkaike Information Criterion (AIC) (20). It is considered that the model with the lowest AIC among other candidate models is one that has sufficient prediction for a given data set (20). The stepwise selection procedure aimed at finding such an optimal model in a systematic manner and this method has been previously used in many biomedical studies $(21,22)$. The forward addition step was started with the null model, which included only the intercept term and the random individual effect. The covariate contributed to the greatest reduction in AIC was then added to the model. The covariates were added one-by-one until the AIC could not be further reduced. Thereafter, the backward elimination step removed the covariate that decreased the AIC. If no further removal of covariates was possible to lower the level of AIC, the elimination step was terminated at this stage. In addition, we compared different proposed models using the average squared prediction errors (PE) for longitudinal data (21). During the process, each of the 209 infants was removed from the estimation sequentially and the corresponding observed
Table 1. Clinical characteristics of the study population

\begin{tabular}{lc}
\hline \multicolumn{1}{c}{ Clinical characteristics } & $\begin{array}{c}\text { No. of infants } \\
(n=209)\end{array}$ \\
\hline GA (wk) & $29.0(26.9-30.7)$ \\
Birth weight (g) & $1175(870-1425)$ \\
Sex (female/male) & $103(49.3 \%): 106(50.7 \%)$ \\
Mode of delivery (vaginal/cesarean section) & $104(49.8 \%): 105(50.2 \%)$ \\
Birth order (singleton/twins) & $169(80.9 \%): 40(19.1 \%)$ \\
Inborn/outborn & $199(95.2 \%): 10(4.8 \%)$ \\
Apgar scores & \\
1 min & $7(6-8)$ \\
5 min & $8(8-9)$ \\
Arterial cord blood & \\
pH & $7.30(7.25-7.34)$ \\
Base deficit (mmol/L) & $-3.8(-6.5$ to -2.2$)$ \\
Prolonged rupture of membrane $>24$ h & $49(23 \%)$ \\
Maternal diabetes & $17(8.1 \%)$ \\
Pre-eclampsia/eclampsia & $30(14.4 \%)$ \\
Antenatal dexamethasone & \\
Infants whose mothers received antenatal & $173(82.8 \%)$ \\
$\quad$ corticosteroids & \\
Cumulative doses (mg) & $24(12-24)$ \\
Time between last dose and delivery (h) & $24(6-118)$ \\
Histologic chorioamnionitis & $64(30.6 \%)$ \\
CRIB score & $1(1-4)$ \\
Worst OI (first 24 h) & $4.6(2.3-7.9)$ \\
Maximum AaDO ${ }_{2}$ gradient (first 24 h) & $95.2(50.3-155.2)$ \\
Respiratory support (first 24 h) & \\
No ventilation & $34(16 \%)$ \\
nCPAP & $36(17 \%)$ \\
IPPV/HFOV & $139(67 \%)$ \\
Age with lowest BP (h) & $5(2-10)$ \\
Lowest systolic BP (mm Hg) & $34(28-40)$ \\
Lowest mean BP (mm Hg) & $26(23-31)$ \\
\hline Rowt diastolic BP (mm Hg) & $21(18-25)$ \\
\hline
\end{tabular}

Results are median (interquartile range) or $\mathrm{n}(\%)$.

$\mathrm{AaDO}_{2}$ gradient, alveolar-arterial oxygen gradient; BP, blood pressure; CRIB, clinical risk index for babies; OI, oxygenation index.

cortisol concentrations were predicted by the estimated model based on 208 infants (i.e. $\mathrm{PE}=$ the average of the leave-one-out errors). The current model gave a PE of 234,341 , whereas the model included only postnatal age had a $\mathrm{PE}$ of 257,753 . The paired leave-one-out prediction errors were assessed by the Wilcoxon signed-rank test, and the result indicated that the current model predicted significantly better than the model with only postnatal age $(p=$ $0.005)$. All tests were performed with the Windows version of S-Plus 2000 (MathSoft Inc., Seattle, WA), and the mixed-effects models were constructed using the function LME.

Ethical approval. The study was approved by the Joint The Chinese University of Hong Kong-New Territories East Cluster Clinical Research Ethics Committee. Written informed consent was obtained from parents.

\section{RESULTS}

Of 238 inborn infants $\leq 32$ wk gestation admitted to the NICU within the 50-mo study period, 199 inborn and 10 outborn infants were longitudinally monitored for serum cortisol concentrations. Of 39 inborn infants not recruited, 6 had chromosomal and/or severe congenital abnormalities, the parents of 21 infants did not wish their infant to participate in the study, and 12 missed the initial recruitment. The overall trend of nonadjusted serum cortisol is illustrated in Figure 1.

In the first stage of analysis, logarithmic transformation of serum cortisol concentrations was performed after its distribution was studied. The covariates being considered in the stepwise selection procedure are listed in the list of parameters 
Table 2. Clinical outcomes of the study population

\begin{tabular}{|c|c|}
\hline Clinical outcomes & $\begin{array}{l}\text { No. of infants } \\
\quad(n=209)\end{array}$ \\
\hline \multicolumn{2}{|l|}{ Cardiovascular } \\
\hline Maximum dose of dopamine $(\mu \mathrm{g} / \mathrm{kg} / \mathrm{min})$ & $5(0-16)$ \\
\hline Cumulative dose of dopamine $(\mu \mathrm{g} / \mathrm{kg})$ & $6830(0-31080)$ \\
\hline Maximum dose of dobutamine $(\mu \mathrm{g} / \mathrm{kg} / \mathrm{min})$ & $0(0-0)$ \\
\hline Cumulative dose of dobutamine $(\mu \mathrm{g} / \mathrm{kg})$ & $0(0-0)$ \\
\hline Maximum dose of epinephrine $(\mu \mathrm{g} / \mathrm{kg} / \mathrm{min})$ & $0(0-0)$ \\
\hline Cumulative dose of epinephrine $(\mu \mathrm{g} / \mathrm{kg} / \mathrm{min})$ & $0(0-0)$ \\
\hline Infants requiring $>1$ vasopressors & $31(14.8 \%)$ \\
\hline Age after birth commenced on vasopressor (h) & $7(4.5-12)$ \\
\hline Duration of vasopressor support (h) & $19(0-55)$ \\
\hline Total volume of crystalloid $(\mathrm{mL} / \mathrm{kg})$ & $42.3(19.8-83.1)$ \\
\hline $\begin{array}{l}\text { Total volume of crystalloid and blood products } \\
(\mathrm{mL} / \mathrm{kg})\end{array}$ & $68.2(29.4-152.9)$ \\
\hline \multicolumn{2}{|l|}{ Respiratory } \\
\hline Duration of IPPV/HFOV (d) & $4(1-14)$ \\
\hline $\begin{array}{l}\text { Duration of positive pressure ventilation } \\
\text { (including nCPAP) (d) }\end{array}$ & $16(5-48)$ \\
\hline Duration of $\mathrm{O}_{2}$ supplementation (d) & $2(1-22)$ \\
\hline Maximum $\mathrm{FiO}_{2}$ & $0.21(0.21-0.35)$ \\
\hline Maximum mean airway pressure $\left(\mathrm{cm} \mathrm{H}_{2} \mathrm{O}\right)$ & $10(7-12)$ \\
\hline $\mathrm{O}_{2}$ requirement at $28 \mathrm{~d}$ of life & $49(23.4 \%)$ \\
\hline $\mathrm{O}_{2}$ requirement at 36 wk postconceptional age & $19(9.1 \%)$ \\
\hline Use of postnatal dexamethasone & $19(9.1 \%)$ \\
\hline Use of postnatal inhaled corticosteroids & $74(35.4 \%)$ \\
\hline Pulmonary interstitial emphysema & $17(8.1 \%)$ \\
\hline Pneumothorax & $2(1.0 \%)$ \\
\hline \multicolumn{2}{|l|}{ Others } \\
\hline $\begin{array}{l}\text { Echocardiographic confirmed patent ductus } \\
\text { arteriosus }\end{array}$ & $78(37.3 \%)$ \\
\hline Patent ductus arteriosus with surgical ligation & $11(5.3 \%)$ \\
\hline Intraventricular hemorrhage $\geq$ grade 3 & $19(9.1 \%)$ \\
\hline Periventricular leukomalacia & $1(0.5 \%)$ \\
\hline Necrotizing enterocolitis & $11(5.3 \%)$ \\
\hline Spontaneous gastrointestinal perforation & $6(2.9 \%)$ \\
\hline $\begin{array}{l}\text { Retinopathy of prematurity requiring laser } \\
\text { therapy }\end{array}$ & $11(5.3 \%)$ \\
\hline Duration of hospitalization (d) & $70(45-111)$ \\
\hline Died & $10(4.8 \%)$ \\
\hline
\end{tabular}

Results are median (interquartile range) or $\mathrm{n}(\%)$.

$\mathrm{FiO}_{2}$, fractional inspired $\mathrm{O}_{2}$ concentration.

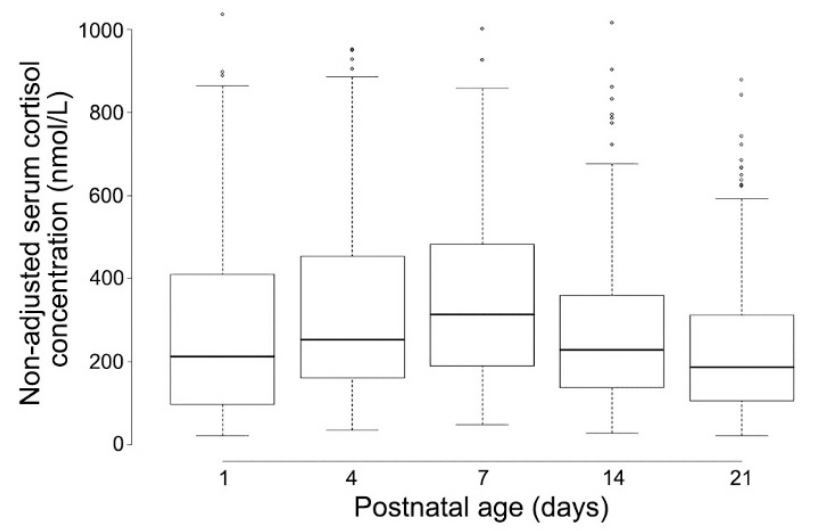

Figure 1. A box and whisker plot describing the distribution of nonadjusted serum cortisol concentrations of preterm infants $\leq 32 \mathrm{wk}$ gestation (at birth) within the first $21 \mathrm{~d}$ of life. The box and whisker plot represents the median, interquartile range, and range. $\mathrm{d} 1, n=190 ; \mathrm{d} 4, n=178 ; \mathrm{d} 7, n=198 ; \mathrm{d} 14$, $n=191 ; \mathrm{d} 21, n=181$.

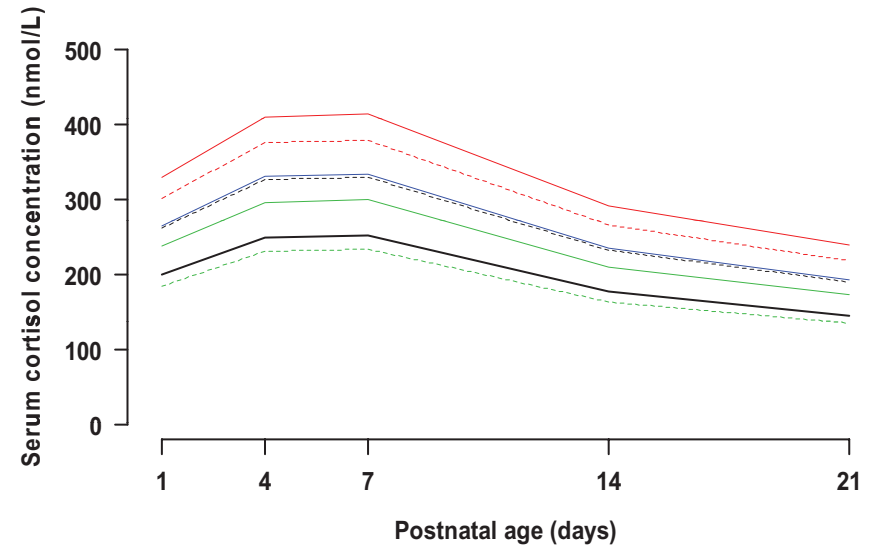

Figure 2. This diagram demonstrates the variation of serum cortisol concentration in preterm infants $\leq 32 \mathrm{wk}$ gestation (at birth) within the first $21 \mathrm{~d}$ of life. The median level (solid black line) was computed by setting an uncomplicated clinical situation as follows: GA $28.7 \mathrm{wk}$ (at birth), no assisted ventilation, no IVH, no requirement for a second vasopressor, no histologic chorioamnionitis, and no severe lung disease at $\mathrm{d} 14$ (i.e. not requiring inhaled corticosteroids). The influence of significant perinatal factors on serum cortisol concentration is also illustrated in the graph. The presence of histologic chorioamnionitis (solid green line), severe lung disease at d 14 (solid blue line), and IVH $\geq$ grade 3 (solid red line), and the use of nCPAP (dotted blue line) and a second vasopressor (dotted red line), all have an effect of shifting the median serum cortisol level (solid black line) upward, whereas the effect of increase in GA (by $1 \mathrm{wk}$; dotted green line) would shift the median serum cortisol level downward. The magnitude of influence of these factors is the degree of shift from the median cortisol level.

(http://links.lww.com/PDR/XXX) that comprised 23 timevarying variables and 58 nontime-varying variables. In the second stage, seven significant factors associated with serum cortisol levels were "forwardly" added to the mixed-effects models in a sequential manner: i) mode of ventilation; ii) intraventricular hemorrhage (IVH) ( $\geq$ grade 3 ); iii) postnatal age; iv) GA; v) treatment with a second vasopressor (i.e. dobutamine); vi) histologic chorioamnionitis; and vii) severe lung disease between 2 and 3 wk of postnatal age associated with the use of inhaled corticosteroids. In the third stage with the "backward" elimination step, none of the aforementioned significant factors could be eliminated from the model. The resultant mixed-effects model indicated that the mean serum cortisol levels were significantly higher on $\mathrm{d} 4(p=0.007)$ and $\mathrm{d} 7(p=0.007)$ but lower on $\mathrm{d} 21(p=0.001)$ compared with the $\mathrm{d} 1$ level (Figs. 1 and 2). The mean serum cortisol concentration was also significantly increased in infants commenced on nasal continuous positive airway pressure (nCPAP) compared with those who were breathing spontaneously without assisted ventilation $(p=0.003)$, whereas infants requiring intermittent positive pressure ventilation (IPPV) or high-frequency oscillatory ventilation (HFOV) did not differ significantly from those who did not require mechanical ventilatory support $(p=$ 0.292 and 0.099). In addition, infants with severe IVH ( $\geq$ grade $3 ; p<0.001$ ), requiring a second vasopressor for blood pressure support $(p<0.001)$, with histologic chorioamnionitis $(p=0.007)$ or with severe lung disease treated with inhaled corticosteroids according to our unit protocol ( $p=0.046)$, all had significantly higher mean serum cortisol concentrations compared with infants who did not develop 
these complications. In contrast, the mean serum cortisol was significantly higher in infants with decreasing GA at birth $(p<0.001)$.

Figure 2 illustrates the estimated median serum cortisol concentration within the first $21 \mathrm{~d}$ of uncomplicated preterm infants born at $28.7 \mathrm{wk}$ gestation, and the positive or negative effects and magnitude of influence of different significant perinatal factors (i.e. factors $\mathrm{i}-\mathrm{ii}$ and $\mathrm{v}-\mathrm{vii}$ ) in shifting the position of this curve. On the basis of above results, we have derived an equation for working out the adjusted cortisol percentile (i.e. the percentile of serum cortisol after taking into account the identified significant perinatal factors) so that neonatologists may evaluate the cortisol response under different clinical circumstances. Details of the equation development and examples for calculation of the adjusted cortisol percentile in different clinical circumstances are provided online (statistical details are available at http://links.lww.com/PDR/XXX). Three cases are described. Patient A had an uneventful clinical course on $\mathrm{d} 1$ and his circulating cortisol was $252 \mathrm{nmol} / \mathrm{L}(9.13 \mu \mathrm{g} / \mathrm{dL})$. The nonadjusted and adjusted cortisol percentiles were 52nd and 72nd, and both were within the normal predicted range. Patient $\mathrm{B}$ was on nCPAP, had severe IVH (grade 4), and required a second vasopressor on d 7. Her serum cortisol was 1,654 $\mathrm{nmol} / \mathrm{L}(59.92 \mu \mathrm{g} / \mathrm{dL})$. The nonadjusted and adjusted cortisol percentiles were 99th and 75th. Her circulating cortisol should not be considered excessive taking into account the stressful clinical situation and corticosteroids supplementation was not indicated for treatment of her systemic hypotension. Patient $\mathrm{C}$ also had an uncomplicated course on d 1 with serum cortisol $40 \mathrm{nmol} / \mathrm{L}(1.45 \mu \mathrm{g} / \mathrm{dL})$. The nonadjusted and adjusted cortisol percentiles were 2 nd and 4 th. The serum cortisol concentration was low and remained $<5$ th percentile after adjustment. Thus, depending on the perinatal factors, the difference between unadjusted percentiles and model-adjusted percentiles could vary from 2 to at least 24 percentiles. In the pair of hypothetical cases with the same GA and circulating cortisol level, the adjusted percentile for the infant with complications and without complications were at the 1st and 20th percentiles, respectively; however, the nonadjusted percentile was 33rd (statistical details are available at http://links.lww.com/PDR/XXX). For ease of use, a web link (http://www.sta.cuhk.edu.hk/pswong/ACortP.html) is provided to enable frontline neonatologists to enter the perinatal factors so that the computer program will automatically use the proposed model to estimate the adjusted percentile from the laboratory-measured serum cortisol concentration.

\section{DISCUSSION}

Our longitudinal results suggest an overall significant increase in serum cortisol level on $\mathrm{d} 4$ and $\mathrm{d} 7$ which then decreases gradually after the first week of life (Fig. 1). The study has also identified seven significant perinatal factors affecting the serum cortisol concentration in preterm infants $\leq 32$ wk gestation during the first $3 \mathrm{wk}$. The most important "intrinsic" factor is GA. The significant "disease-related" factors are histologic chorioamnionitis and severe IVH ( $\geq$ grade 3 ), whereas the significant "treatment-related" factors are the use of nCPAP, a second vasopressor, and severe lung disease necessitating the use of inhaled corticosteroids in accordance to our unit protocol. More importantly, the statistical model enables us to derive an equation for working out the adjusted cortisol percentile after correction for significant perinatal stressful events. This equation is available on our Web site and the adjusted percentile can be easily calculated during the routine clinical round. Frontline neonatologists can now logically interpret circulating cortisol levels so that the resultant percentile value may be appropriately evaluated along with the clinical features, both in ill and healthy preterm infants. The use of "adjusted cortisol percentile" could assist clinicians to decide whether a symptomatic infant has an inadequate cortisol response and who might benefit from corticosteroid replacement.

As far as we are aware, no previous study has established the "normative" range of serum cortisol or "perinatal factoradjusted cortisol percentiles" for preterm infants $\leq 32 \mathrm{wk}$ gestation. One of the major obstacles is that by definition there are no "normal" preterm infants. More importantly, postnatal serum cortisol concentration may change physiologically with postnatal age $(1,2)$, and the hormone level may be affected positively or negatively by both intrinsic and extrinsic perinatal factors (14). Previous attempts by us $(5,10)$ and other investigators (23) did not take into account of such factors. One of the most appropriate methods for assessing serum cortisol concentrations of a heterogeneous group of patients is to study a well-defined population and to adjust the hormonal level by identifying the significant influential factors. It is, however, not possible to plot a mathematically adjusted circulating cortisol value on a conventional two-dimensional percentile chart because the many influential factors identified would require a multidimensional chart to accurately describe the position of a fully adjusted value. Such a format of presentation would be extremely difficult to interpret and impracticable to use in the daily hospital setting. Alternatively, we can offer $2^{5} \times 4$ (i.e. including four modes of ventilation) $=128$ three-dimensional percentile plots (i.e. GA as an extra dimension) representing 128 different scenarios, but again this method of presentation would be very confusing and clumsy. Hence, the most simple and accurate way is to provide a web link for entering data of significant factors and to allow the program to compute the adjusted cortisol percentile. The final result is, thus, tailored for a specific infant with specific clinical features at a specific timepoint.

A potential problem associated with this type of study design is the lack of a comprehensive data set. We have taken stringent precautions to avoid this shortcoming. Our data were collected prospectively, and the information was standardized and accurately monitored. More importantly, we had collected 81 variables in the initial data set for statistical analysis and are confident that the majority of important perinatal factors have been included for vigorous scrutiny. Another crucial feature is the longitudinal nature of serum cortisol monitoring, which included three timepoints in the most vulnerable period during the first week of life. Second, the larger the study population, the higher would be the chance of identifying the significant perinatal factors. A cohort of 209 preterm infants 
may be considered as a moderately large number for proposing the structure of an initial statistical model, especially with longitudinal monitoring of serum cortisol concentrations at five different timepoints. In a separate analysis, we have also included 33 infants with GA ranging from 33 to $35 \mathrm{wk}$ (admitted within the same study period), and the same seven significant perinatal factors were identified by the statistical analysis (AIC). In addition, we have used a similar but alternative statistical technique, the Bayesian Information Criterion (BIC) (24), for cross referencing of outcomes. The BIC method is well documented to be conservative and would usually include fewer significant factors into the model. The outcome of the BIC selection indicated that the first five significant factors selected by the AIC method were also included in the BIC model, and the latter model excluded only histologic chorioamnionitis and severe lung disease associated with the use of inhaled corticosteroids at d 14 as significant factors.

The critical perinatal factors identified in this cohort were all stressful events. Severe IVH is a potent stressful complication that has been recently reported to be associated with high circulating cortisol levels (9). Chorioamnionitis, an infective/ inflammatory process, has been associated with increased serum cortisol level in VLBW infants (25). However, the association between increased cortisol and nCPAP was unexpected. There are several plausible explanations. The routine use of narcotic analgesia, such as morphine or fentanyl, for heavy sedation of intubated infants (i.e. IPPV and HFOV) could have accounted for the partial alleviation of stress in ventilated infants $(26,27)$. In addition, infants on nCPAP would not be sedated and were almost always prescribed caffeine for prevention of apnea and facilitation of respiration. The increased work of breathing for patients on nCPAP could be associated with higher serum cortisol levels. In contrast, increased severity of RDS requiring mechanical ventilation was unlikely the reason for lower cortisol levels because low levels were also observed in infants who had normal lungs and did not require any ventilatory support. In addition, although a proportion of younger gestation infants and infants with refractory hypotension have been described to have adrenal insufficiency with very low circulating cortisol (3-5), the overall findings in this cohort suggested that the majority of younger gestation infants and those requiring a second vasopressor for blood pressure support were probably more stressed and had significantly higher circulating cortisol levels than older gestation infants who required none or only one vasopressor. Thus, our results provide evidence to support the hypothesis that the HPA axis of most preterm infants is able to mount a measurable cortisol response to stressful stimuli. Whether the magnitude of the response was adequate in every case for coping with the stressful situation was not certain. Our findings also suggested that infants with severe lung disease at d 14 were highly stressed. The latter observation was in accordance with our previous findings that VLBW infants who developed BPD were more stressed and had significantly higher serum cortisol levels on d 14 than those who did not subsequently develop BPD (18).
There are limitations in this study. First, our formula could only give an adjusted cortisol percentile after correction of significant perinatal factors, but it could not indicate whether the cortisol level represented adequate HPA axis response for a specific clinical situation. Hence, the adjusted cortisol percentile provides additional information, but frontline neonatologists are still required to interpret the results in conjunction with the clinical picture. Second, of the significant factors selected, severe lung disease at $\mathrm{d} 14$ was a relatively late event and the clinical definition was only arbitrarily defined. It was also the least significant factor (7th factor) among others chosen into the model. This factor could not be taken into account before the first $2 \mathrm{wk}$ of life. However, the significantly higher serum cortisol level in these patients represented a genuine adrenocortical response to stress, as we had previously demonstrated that prolonged use of inhaled corticosteroid could suppress the HPA axis.(28) Third, as this was a single center study, it is plausible that some of the clinical factors identified could theoretically elicit different cortisol responses under different clinical circumstances in other centers. For example, as only one type of nCPAP was used during the study period, infants at other centers employing different types of nCPAP could respond differently. Finally, the modeladjusted percentiles should be interpreted with caution and considered as arising from an initial preliminary study. We also caution the interpretation of adjusted percentiles at the extremes (i.e. close to 0 or 100 percentile) as their variability would be more pronounced. Additional research is required to verify this initial model and to assess the precision of the adjusted percentiles.

In conclusion, we have identified seven factors that can significantly affect the serum cortisol concentration in early postnatal life. As most of these factors are stressful events that are associated with increased circulating cortisol levels, this study provides good evidence that the majority of preterm infants are able to produce a measurable cortisol response at time of stress. Furthermore, the recognition of significant factors allows an adjusted cortisol percentile to be estimated via an easily accessible web link. Frontline neonatologists may have more meaningful and better interpretation of circulating cortisol results after taking into account different clinical events. Our results could potentially allow identification of clinical situations where corticosteroids replacement would be beneficial (e.g. refractory hypotension with adjusted cortisol percentile $<25^{\text {th }}$ percentile) but more importantly, avoidance of excessive or indiscriminate use of systemic corticosteroids where the objective evidence does not indicate adrenocortical insufficiency (e.g. refractory hypotension with adjusted cortisol percentile $>50$ th percentile). In the latter situation, other important causes such as hypovolemia, high positive pressure ventilation, and septic shock must be identified. The next step is to conduct a large-scale prospective study to verify the proposed statistical model and to further evaluate the clinical usefulness of the adjusted cortisol percentile in a day-to-day clinical setting for the management of preterm, surgical, or critically ill infants, who are most at risk of adrenocortical insufficiency. 


\section{REFERENCES}

1. Ng PC 2000 The fetal and neonatal hypothalamic-pituitary-adrenal axis. Arch Dis Child Fetal Neonatal Ed 82:F250-F254

2. Mesiano S, Jaffe RB 1997 Developmental and functional biology of the primate fetal adrenal cortex. Endocr Rev 18:378-403

3. Helbock HJ, Insoft RM, Conte FA 1993 Glucocorticoid-responsive hypotension in extremely low birth weight newborns. Pediatrics 92:715-717

4. Ng PC, Lam CW, Fok TF, Lee CH, Ma KC, Chan IH 2001 Refractory hypotension in preterm infants with adrenocortical insufficiency. Arch Dis Child Fetal Neonatal Ed 84:F122-F124

5. Ng PC, Lee CH, Lam CW, Ma KC, Fok TF, Chan IH, Wong E 2004 Transient adrenocortical insufficiency of prematurity (TAP) and systemic hypotension in very low birth weight infants. Arch Dis Child Fetal Neonatal Ed 89:F119-F126

6. Watterberg KL, Gerdes JS, Cook KL 2001 Impaired glucocorticoid synthesis in premature infants developing chronic lung disease. Pediatr Res 50:190-195

7. Huysman MW, Hokken-Koelega AC, De Ridder MA, Sauer PJ 2000 Adrenal function in sick very preterm infants. Pediatr Res 48:629-633

8. Watterberg KL, Gerdes JS, Cole CH, Aucott SW, Thilo EH, Mammel MC, Couser RJ, Garland JS, Rozycki HJ, Leach CL, Backstrom C, Shaffer ML 2004 Prophylaxis of early adrenal insufficiency to prevent bronchopulmonary dysplasia: a multicenter trial. Pediatrics 114:1649-1657

9. Aucott SW, Watterberg KL, Shaffer ML, Donohue PK, PROPHET Study Group 2008 Do cortisol concentrations predict short-term outcomes in extremely low birth weight infants? Pediatrics 122:775-781

10. Ng PC, Lam CW, Lee CH, Ma KC, Fok TF, Chan IH, Wong E 2002 Reference ranges and factors affecting the hCRH test in preterm, very low birth weight infants. J Clin Endocrinol Metab 87:4621-4628

11. Heckmann M, Hartmann MF, Kampschulte B, Gack H, Bödeker RH, Gortner L, Wudy SA 2005 Cortisol production rates in preterm infants in relation to growth and illness: a noninvasive prospective study using gas chromatography-mass spectrometry. J Clin Endocrinol Metab 90:5737-5742

12. Lee MM, Rajagopalan L, Berg GJ, Moshang T Jr 1989 Serum adrenal steroid concentrations in premature infants. J Clin Endocrinol Metab 69:1133-1136

13. Hingre RV, Gross SJ, Hingre KS, Mayes DM, Richman RA 1994 Adrenal steroidogenesis in very low birth weight preterm infants. J Clin Endocrinol Metab 78:266270

14. Ng PC 2008 Is there a 'normal' range of serum cortisol concentration for preterm infants? Pediatrics 122:873-875
15. Howie RN, Liggins GC 1982 The New Zealand study of antepartum glucocorticoid treatment. In: Farrell PM (ed) Lung Development: Biological and Clinical Perspectives, Vol II. Academic Press, New York, pp 255-265

16. Ment LR, Oh W, Ehrenkranz RA, Philip AG, Vohr B, Allan W, Duncan CC, Scott DT, Taylor KJ, Katz KH, Schneider KC, Makuch RW, Philip AG 1994 Low-dose indomethacin and prevention of intraventricular hemorrhage: a multicenter randomized trial. Pediatrics 93:543-550

17. Ballard JL, Khoury JC, Wedig K, Wang L, Eilers-Walsman BL, Lipp R 1991 New Ballard score, expanded to include extremely premature infants. J Pediatr 119:417423

18. Ng PC, Lee CH, Lam CW, Fok TF, Chan IH, Ma KC, Wong E 2004 Early pituitary-adrenal response and respiratory outcomes in preterm infants. Arch Dis Child Fetal Neonatal Ed 89:F127-F130

19. Goldstein H 1995 Multilevel statistical models. In: Goldstein H (ed) Kendalls Library of Statistics 3. Edward Arnold, London, pp 87-94

20. Lai TL, Shih MC, Wong SP 2006 A new approach to modeling covariate effects and individualization in population pharmacokinetic. J Pharmacokinet Pharmacodyn 33:49-74

21. Lai TL, Shih MC, Wong SP 2006 Flexible modeling of fixed and random effects in generalized mixed models for longitudinal data. Biometrics 62:159-167

22. Akaike H 1974 A new look at the statistical model identification. IEEE Trans Automat Contr 19:716-723

23. Heckmann M, Wudy SA, Haack D, Pohlandt F 1999 Reference range for serum cortisol in well preterm infants. Arch Dis Child Fetal Neonatal Ed 81:F171-F174

24. Konishi S, Kitagawa G 2008 Bayesian information criteria. In: Konishi S and Kitagawa G (eds) Information criteria and statistical modeling. Springer, New York, pp 211-236

25. Watterberg KL, Scott SM, Naeye RL 1997 Chorioamnioitis, cortisol, and acute lung disease in very low birth weight infants. Pediatrics 99:E6

26. Quinn MW, Wild J, Dean HG, Hartley R, Rushforth JA, Puntis JW, Levene MI 1993 Randomised double-blind controlled trial of effect of morphine on catecholamine concentrations in ventilated pre-term babies. Lancet 342:324-327

27. Aloisi AM, Aurilio C, Bachiocco V, Biasi G, Fiorenzani P, Pace MC, Paci V, Pari G, Passavanti G, Ravaioli L, Sindaco G, Vellucci R, Ceccarelli L 2009 Endocrine consequences of opioid therapy. Psychoneuroendocrinology 34:S162-S168

28. Ng PC, Fok TF, Wong GW, Lam CW, Lee CH, Wong MY, Lam K, Ma KC 1998 Pituitary-adrenal suppression in preterm, very low birth weight infants after inhaled fluticasone propionate treatment. J Clin Endocrinol Metab 83:2390-2393 